\title{
Corela
}

Cognition, représentation, langage

HS-33 | 2020

Textuel, textiel. Repenser la textualité numérique

\section{Présentation. Les devenirs du texte numérique natif}

Ingrid Mayeur et Marie-Anne Paveau

\section{(2) OpenEdition}

1 Journals

\section{Édition électronique}

URL : https://journals.openedition.org/corela/11749

DOI : 10.4000/corela. 11749

ISSN : 1638-573X

Éditeur

Cercle linguistique du Centre et de l'Ouest - CerLICO

Référence électronique

Ingrid Mayeur et Marie-Anne Paveau, «Présentation. Les devenirs du texte numérique natif », Corela [En ligne], HS-33 | 2020, mis en ligne le 23 septembre 2020, consulté le 13 juillet 2021. URL : http:// journals.openedition.org/corela/11749; DOI : https://doi.org/10.4000/corela.11749

Ce document a été généré automatiquement le 13 juillet 2021.

\section{(c) (i) (2)(2)}

Corela - cognition, représentation, langage est mis à disposition selon les termes de la licence Creative Commons Attribution - Pas d'Utilisation Commerciale - Partage dans les Mêmes Conditions 4.0 International. 


\title{
Présentation. Les devenirs du texte numérique natif
}

\author{
Ingrid Mayeur et Marie-Anne Paveau
}

1 Dans son ouvrage La linguistique textuelle, Jean-Michel Adam proposait de définir la textualité comme « un ensemble d'opérations qui amènent un sujet à considérer à la projection et/ou à la lecture/audition qu'une suite d'énoncés forme un tout signifiant » (2011 : 14). Le texte est donc ce qui, aux yeux du lecteur, constitue une unité de sens cohérente. Au stade le plus récent de sa réflexion, qui porte désormais sur le «faire texte ", il préfère à la définition une interrogation sur « les facteurs qui amènent un sujet écrivant ou lisant à porter sur une suite d'énoncés un jugement de textualité et sur les conséquences de ce jugement sur l'interprétation des énoncés » (Adam, Philippe 2015 : 45). Ce jugement, précise-t-il juste après, repose sur « un triple sentiment », de connexité, de cohésion et de cohérence. Cependant, compte tenu des spécificités des nouveaux médias, et notamment des réseaux sociaux, peut-on encore considérer que ces trois sentiments reposant sur les opérations de textualisation traditionnelles (reprise anaphorique, liages textuels, etc.) permettent de «faire texte» (Adam dir. 2015) dans un environnement numérique ? C'est la question générale qui a guidé la constitution de ce dossier, consacré à l'exploration de la dimension proprement numérique des processus de textualisation dans les écosystèmes connectés, ce que veut dire natif dans l'expression texte numérique natif: le terme désigne dans cette perspective des textes produits originellement au sein d'espaces connectés, et désigne des textualités, ou, on le verra, des textialités, qui n'existent pas hors de ces espaces.

\section{Le textiel. Une notion pour penser la textualité connectée}

2 Les médias informatisés, c'est-à-dire, pour aller vite, les espaces de production de texte en ligne ${ }^{1}$, qu'ils s'agisse de sites, de blogs, de journaux ou de réseaux sociaux, sont désormais envisagés comme des machines textuelles (Jeanneret et Souchier 2005, Tardy et Jeanneret 2007). Ils conditionnent l'énonciation éditoriale des textes (Souchier 1998), 
strate énonciative qui configure l'énonciation linguistique et inscrit les textes dans des standards prédéfinis par les affordances technologiques des espaces d'écriture ( $\underline{\mathrm{CMS}}$, $\underline{\mathrm{API}}$. Ces médias présentent la particularité d'être à la fois outils de lecture et d'écriture des textes; ils requièrent la participation active du lecteur et les gestes de lecture du texte (défilement, activation des hyperliens, ouverture de fenêtres, etc.) se font également gestes d'écriture, qui en définissent la structuration. D'où une expérience individuelle du texte, susceptible d'être actualisé différemment selon les lecteurs et leurs pratiques: l'écriture et la lecture en ligne s'articulent étroitement dans un procédé d'écrilecture, selon le terme proposé par Barbosa 1992 (voir Paveau 2017a, article "Écriture numérique").

La dimension opératoire (c'est-à-dire dynamique, plurisémiotique et manipulatoire) du texte numérique natif rend sans doute, pour partie, obsolètes les traits qui fondent la cohérence textuelle dans l'univers de l'imprimé, et, partant, les critères définissant le jugement de textualité. Le recours à la notion de textiel, qui tient compte du caractère à la fois langagier et manipulable du texte en ligne, nous apparaît alors pertinent pour questionner le devenir du textuel dans son environnement numérique natif, et tenter une saisie des opérations de textualisation qui s'y nouent.

Depuis le début des années 1990, les recherches en Sciences de l'information et de la communication (SIC) ont pris pour objet la circulation des connaissances dans l'environnement numérique. La discipline interroge en effet cette circulation sous l'angle de la matérialité des artefacts qui interviennent dans le procès de communication; elle fait ainsi figure de pionnière dans les apports théoriques entourant la textualité numérique. Dès 1993, l'historien Roger Chartier propose d'appréhender les mutations du texte à l'écran dans la continuité de celles engendrées par d'autres supports d'écriture/lecture que sont le codex, le volumen et le livre imprimé (Chartier 1993, 2001). Les recherches d'Emmanuël Souchier (1996) sur les écrits d'écran mettent en lumière les transformations de l'acte d'écriture/lecture par les outils informatiques qui conditionnent désormais l'accès au texte : perte de maitrise du scripteur sur l'ensemble du processus d'écriture, généralisation du défilement vertical, possibilité de multiplication des fenêtres dynamisant les pratiques de lecture, médiation de l'écran qui ne laisse entrevoir qu'une partie des composantes du texte, etc.

L'ouvrage collectif dirigé par Jean Davallon, Marie Després-Lonnet, Yves Jeanneret, Joëlle Le Marec et Emmanuël Souchier, intitulé Lire, écrire, récrire (2003), ouvre quelques années plus tard une réflexion sur les liens unissant usage, texte et technique : le texte numérique y est désormais considéré en tant que textiel, objet tout à la fois langagier et technique, manipulable, inscrit dans un réseau de relations. Y est envisagé notamment un "textiel de communication" qui tire le texte vers une véritable pratique en contexte numérique. Se posent dès lors les questions suivantes: si, comme l'a relevé Roger Chartier $(1997,2006)$, les textes en ligne tendent à se dissoudre dans un continuum, si leur hétérogénéité est partiellement occultée par l'homogénéité des dispositifs de réception et la faible généricité qu'ils autorisent, comment cerner les frontières de la forme-texte, son organisation, et quels sont les modes d'appréhension qui guident les usages d'écriture/lecture? C'est l'approche sémiotique, centrée sur l'activité interprétative du lecteur, qui est alors convoquée à travers une expérience concrète de consultation de sites consacrés aux OGM ; elle révèle que l'actualisation de la formetexte s'effectue en fonction de la prédilection sémiotique du lecteur, qui met à 
contribution sa propre mémoire des formes pour les qualifier et leur donner du sens (Souchier et al. 2003).

6 Par la suite, la notion de textiel sera encore étudiée à l'occasion d'un numéro de la revue Communication et langage (Souchier dir., 2004), où elle se profile comme un prolongement du document numérique, doté de possibilités dynamiques et opératoires. Plus récemment, le premier numéro de la revue Études digitales (Cormerais et Gilbert dirs., 2016), intitulée Le texte à venir, a offert une série de contributions interrogeant, à travers le devenir du texte dans l'environnement connecté, la manière dont le numérique agissait sur la culture, les modes de pensée, voire l'activité du chercheur. Revenant à cette occasion sur ses travaux à la faveur d'un grand entretien ${ }^{2}$, Emmanuël Souchier signalait alors la «textualisation de notre rapport au monde» sous l'effet de l'informatisation de « la quasi-totalité des pratiques sociales d'élaboration, de création, de production, de circulation des biens ou activités matériels et symboliques " (Souchier 2016: 205). La textualité numérique se fait ainsi vecteur d'une culture à l'intersection du computationnel et du symbolique, du machinique et de l'humain.

\section{Le numérique natif dans les sciences du texte. Absences et verrous}

7 Ainsi que le montre la genèse du concept, inscrite dans les recherches en SIC, le textiel est utilisé pour rendre compte de la complexification du texte appréhendé comme document et comme support de transmission des connaissances, dans l'environnement numérique. Dans cette perspective, la saisie de la forme-texte est orientée vers l'appropriation de l'information en vue d'un usage futur. Toutefois, si les matériaux langagiers peuvent être étudiés comme documents, ils peuvent également l'être en tant que textes ou discours, ainsi que le rappelle Marie-Anne Paveau $(2015 \mathrm{~b}, \$ 3)$ à la suite de Jean-Michel Adam (2011) dans un numéro que la revue Itinéraires. littérature, textes, cultures consacrait aux « Textualités numériques ». L'auteure pose alors le constat d'une timide prise en compte du numérique dans le domaine de l'analyse du discours, mais de sa quasi-absence dans celui de la linguistique textuelle.

8 Si le numérique est désormais intégré à presque tous les travaux en sciences humaines et sociales, il reste en effet aux portes de certains domaines des sciences du langage, dont la linguistique textuelle et les recherches sur l'écriture, et particulièrement sous ses formes nativement numériques (Paveau 2015a). Textualistes et spécialistes de l'écriture tardent en effet à reconnaître et intégrer la conversion numérique de l'écriture des textes informatisés, comme le montrent des synthèses récentes sur ces questions, dans le domaine francophone en tout cas, où la dimension numérique semble introuvable ou réduite à la portion congrue (par exemple Lefebvre et al. dir. 2016, Cislaru, Olive 2018, Lieutard (org.), 2019). Le récent ouvrage de Cislaru et Olive sur les processus de textualisation, par exemple, porte l'attention sur la production du texte au cours du processus d'écriture, à partir des logiciels de suivi de rédaction. Ce faisant, les auteurs se proposent de porter « un regard linguistique sur un nouvel objet d'analyse, les jets textuels, i.e. les séquences langagières produites en un seul jet pendant le processus de rédaction, en décrivant leur structure et leur rôle dans le processus de textualisation. » (Cislaru et Olive 2018: 9). S'il s'agit bien ainsi, au sens strict, d'observer les processus de textualisation à partir de textes produits dans un environnement numérique (ici, la production de travaux universitaires de Master 1 ou 
2), le logiciel est mobilisé en tant qu'il permet de conserver une mémoire des pauses ou des repentirs du scripteur et, de ce fait, de matérialiser les strates d'une écriture en cours; les auteurs observent d'ailleurs, de ce point de vue, que l'écriture numérique facilite les retours sur le texte (Ibid.: 26). Toutefois, ces analyses ne portent pas sur les composantes relationnelles, techniques et opératoires des énoncés numériques tels qu'ils s'élaborent dans les environnements connectés, et, partant, ne concernent pas les opérations de textualisation qui se jouent en ligne à travers l'actualisation d'une forme textuelle par l'écrilecteur.

En linguistique, la problématique du texte numérique est en effet essentiellement traitée à travers les questions que posent la numérisation des textes et leur traitement automatique dans le cadre du travail sur les grands corpus, dans le domaine de la linguistique de corpus ${ }^{3}$. Ayant pour objectif le traitement automatique des énoncés, elle a intégré la donne du numérique dès les années 1990. L'objet de la démarche vise la constitution de corpus fiables, à partir de l'opération d'extraction : comme le précise François Rastier, la linguistique de corpus fragmente et délinéarise ${ }^{4}$ le texte, le reclassant en catégories pour mieux en rendre compte (Rastier 2007, 2011). C'est une linguistique qui travaille essentiellement sur des textes originellement imprimés puis numérisés, rarement sur des productions en ligne; quand c'est le cas, c'est la fragmentation et le classement des éléments qui prédominent, dans l'objectif de construire une interprétation qui serait invisible à l'œil, mais rendue explicite par l'instrument (Habert 2005), et non la volonté de rendre compte des modes de production-réception de textes produits dans des interfaces informatiques.

10 La question du texte numérique reste, dans ce type de perspective (celle de la sémantique textuelle ou de la sémiotique des textes) arrimée à celle d'un texte conçu sur le modèle des écrits prénumériques. C'est par exemple le cas chez Rossana De Angelis, qui traite le texte numérique depuis le cadre de la numérisation des livres et traitant par conséquent de la transposition des traits du texte imprimé sur support numérique (De Angelis 2018). Le texte numérique est dans cette approche une forme particulière et restreinte, c'est-à-dire le texte imprimé numérisé, qui conserve son intégrité en tant que texte. Le terme transposition est tout à fait significatif d'une perspective non écosystémique bien différente de celle qui est adoptée dans le présent numéro: les dispositifs numériques sont observés comme outils pour lire, extraire, étudier, etc., et non pas comme lieu de l'écriture et environnement de la production textuelle. De fait, dans ce type de travaux, le texte numérique natif, ou textiel, texte qui réinvente la textualité en textialité à partir des interfaces d'écrilecture connectée, en recréant de nouvelles manières de « faire texte », n'est pas envisagé.

11 Le texte en ligne, c'est-à-dire, répétons-le, produit dans un espace connecté à travers des programmes d'écriture, qui est pourtant devenu une forme aussi fréquente qu'ordinaire, pérennisée à travers les différents avatars historiques du web (forums, blogs, réseaux sociaux, médias sociaux), reste donc encore extérieur aux sciences du langage. Par contraste, il est largement traité du point de vue communicationnel et également littéraire (voir section suivante).

Que la linguistique textuelle, ou les sciences du texte si l'on veut élargir l'empan disciplinaire, ne prennent toujours pas en compte la textualité numérique native, alors que la notion de texte est évidemment définitoire de leurs approches, théories et méthodes, ne peut que s'expliquer; nous voulons dire qu'il ne s'agit pas du hasard des développements des recherches et qu'il doit donc exister des verrous, produits par la 
conception de la discipline et de ses objets, qui ont empêché et empêchent encore ces disciplines d'intégrer à leurs cadres les caractéristiques des textualités numériques natives. Ces verrous nous semblent tenir à l'épistémè des sciences du langage, à une forme de conservatisme disciplinaire, à des positions idéologiques sur le texte et plus largement la littéracie, et à une méconnaissance des univers numériques connectés.

13 - Verrou épistémologique. Force est de constater que les sciences du langage, dans les aires internalistes qui en constituent encore les formes les plus légitimes, mais aussi souvent dans leurs aires les plus contextualistes et constructivistes (linguistique textuelle, analyse du discours, interactionnisme et sociolinguistique) restent attachées à des modèles dualistes qui isolent le langagier de ses dimensions sociales, matérielles ou culturelles, subsumées sous l'étiquette extralinguistique. La prise en compte des énoncés numériques natifs est, on l'a dit, largement dépendante de la linguistique de corpus qui vise un traitement automatique écartant de fait l'étude écologique ${ }^{5}$. On a vu plus haut qu'elle portait généralement sur des textes imprimés numérisés pour l'analyse et donc exportés dans des environnements numériques logiciels. Quand elle porte sur des énoncés en ligne, c'est principalement sur les réseaux sociaux et spécifiquement sur Twitter que les linguistes jettent leur dévolu: le dispositif de la plateforme et le format réduit de la fenêtre d'écriture (140 puis 180 signes) autorise en effet la collecte aisée d'un nombre important d'items permettant de maintenir la méthode de l'extraction et de la normalisation des énoncés en vue de leur traitement; dans cette perspective, la dimension textuelle n'est pas prise en compte (voir notamment les travaux du Consortium CORLI). La question du «faire texte», des formes que peuvent prendre le jugement de textualité dans les textes numériques natifs, n'est pas encore posée, faute d'une conception du texte qui dépasserait la distinction entre composantes linguistiques et techniques pour envisager une seule unité, dans laquelle ils seraient coconstitutifs.

14 - Verrou disciplinaire. Au verrou épistémologique est lié un verrou disciplinaire qui est sans doute la nature de l'objet de la linguistique : le maintien de la distinction matière/ langage s'explique par la nature langagière stricto sensu de l'objet des sciences du langage, qu'il s'agisse du mot, de la phrase, du texte ou du discours. La matérialité, qu'elle soit sociale, historique, idéologique, etc., ou qu'elle constitue une matière physique (papier, pierre, objets, etc., ou, en ce qui nous concerne ici, logicielle) n'est pas considérée comme appartenant à l'ordre du langage. L'intégrer serait modifier la discipline et courir par là le risque de sa disparition. Mais l'objet des sciences du langage est modifiable, comme ceux de toutes les disciplines d'ailleurs, parce qu'il est inscrit dans les réalités évolutives et mouvantes de l'histoire. Il n'y a donc pas de raison pour que l'objet défini à la fondation de la linguistique comme science il y a plus d'un siècle soit conservé dans son intégrité logocentrée d'époque.

15 - Verrou idéologique. Ni l'épistémologie ni la disciplinarité n'échappent à l'idéologie et aux autres composants de la culture (choix politiques, options morales, considérations esthétiques, etc.). Les univers numériques font l'objet depuis leur apparition de discours polémiques et de positions tranchées, technophiles ou technophobes. Il se trouve qu'une figure linguistique de premier plan, Noam Chomsky, défend depuis longtemps des positions particulièrement technophobes, à propos de Twitter notamment qu'il accuse d'appauvrir l'écriture et la langue (Chomsky 2010, 2011). Et sur la question du texte précisément, l'ouvrage de Rafaele Simone par exemple, linguiste italien d'une certaine importance, défend l'idée que les textes en ligne n'en sont pas, ne 
satisfaisant pas aux critères de textualité, essentiellement à cause de leur éparpillement, de leur diffraction et de leur pauvreté (Simone 2012). Cette approche préthéorique et polémique des écritures natives sur les blogs et surtout les réseaux sociaux n'est pas isolée, et rejoint une méfiance relativement partagée envers les pratiques scripturales numériques natives.

16 - Verrou pratique. Enfin, la méconnaissance des outils informatiques permettant l'écriture en ligne (API, CMS) et les pratiques numériques natives restreintes voire parfois inexistantes des linguistes constituent également une explication à cette distance par rapport aux écritures natives. Particulièrement, l'idée que nous ne sommes plus le seul sujet scripteur quand nous écrivons en ligne reste encore contreintuitive et difficile à admettre chez les spécialistes du langage, qui gardent souvent la conception d'un sujet maîtrisant ses productions. Mais, comme le montrent bien Cléo Collomb et Samuel Goyet dans ce numéro, les machines écrivent bien avec nous et même, aussi difficile qu'il soit de le penser, à notre place. Ce verrou pratique peut encore être compris au sens de méthodologique: comment, pratiquement, aborder ces corpus composites et polyphoniques, et avec quels critères de délimitation? Comment combiner approche écosystémique des discours numériques et nécessaire stabilisation des énoncés à des fins d'analyse ? Des propositions méthodologiques originales ont été émises, à l'instar de celle de Laetitia Émerit mobilisant la notion de lieu de corpus comme espace numérique délimité intégrant l'instabilité, la mixité et l'incomplétude constitutives des énoncés numériques natifs (Émerit 2016). Au demeurant, la question est rarement abordée de front, et il semble difficile de trouver un équilibre satisfaisant entre l'étude de cas en contexte mais nécessairement contingente, et l'extraction menant à la constitution d'un corpus (de tweets, de posts, etc.) analysable, n'autorisant pas cependant la conservation de l'environnement des textes.

\section{Des propositions pour l'analyse des textes numériques natifs}

17 Ce dossier sur la textualité-textialité numérique est issu de nos interrogations, recherches et travaux sur les dimensions concrètes de la matérialité informatique du texte numérique natif, et leurs conséquences sur la redéfinition du texte et les orientations théoriques qu'elle implique. Nous présentons ici nos réflexions en sciences du langage, accompagnées d'un certain nombre de travaux récents sur les discours numériques natifs, et rappelons les propositions faites depuis les années 1990 en SIC et littérature, ainsi que des travaux en didactique et dans le domaine du livre et de l'édition.

\subsection{Sciences du langage}

Dans le cadre de l'analyse du discours numérique, et à partir de la notion de technodiscours (discours numérique natif coconstitué d'éléments langagiers et technologiques), Marie-Anne Paveau a fait plusieurs propositions pour rendre compte des spécificités des textes numériques (2013, 2015b, 2017a). Les six traits définitoires des technodiscours (composition, délinéarisation, augmentation, relationalité, investigabilité et imprévisibilité) lui ont permis de décrire des formes textuelles modifiées par les environnements connectés, autrement dit textialisées. La 
délinéarisation, en particulier, opérée par les liens hypertextuels (Paveau 2016), reconfigure la textualité de manière importante en fusionnant les actes d'écriture et de lecture (l'écrilecture) et en faisant du texte une matière à manipuler; de ce fait les dispositifs de cohésion textuelle sont également affectés. Ces caractéristiques l'ont amenée à redéfinir la notion d'observable dans les corpus en ligne (Paveau 2017b), en suggérant de passer métaphoriquement de la méthode du microscope (observation de corpus fixés, à la main ou à l'instrument) à celle de la caméra subjective (observation de corpus subjectifs, dynamiques et évolutifs).

Ingrid Mayeur, qui a posé la question du textiel dans le double champ des SIC et des sciences du langage (Mayeur 2016), a interrogé la textialité des discours scientifiques produits sur les carnets de recherche dans sa thèse de doctorat, Recherches en cours. Discours de savoir numériques et actualité scientifique dans les carnets de recherche de la plateforme Hypothèses (Mayeur 2019). Son exploration des formes de la matérialité textuelle en ligne, menée à partir des formes de l'énonciation éditoriale, de l'examen de la généricité des textes, de la description des gestes d'élaboration du savoir (gestes discursifs et gestes de la recherche), ou encore de l'hypertextualité (Mayeur 2018), permet de repenser la textualité scientifique à partir de la double référence théorique des sciences de l'information et de la communication et de l'analyse du discours.

D'autres travaux récents en analyse du discours et du texte ont alimenté l'horizon épistémologique et théorique de cette livraison de Corela, notamment les deux collectifs sur l'hypertexte dirigés par Justine Simon (Simon dir. 2016 et 2018) et les propositions de Laetitia Émerit précédemment mentionnées. On le voit, c'est la question des observables et de leur stabilisation qui a guidé les premières investigations sur la circonscription des textes et discours numériques.

21 Peu de collectifs ont été consacrés précisément à cette question du devenir textiel du texte numérique, pourrait-on dire de manière un peu deleuzienne. Un numéro de la revue Itinéraires, entièrement consacré à cette question "Textualités numériques ", paraît en 2015 (Paveau dir. 2015) ; en 2016 parait au Brésil un numéro bilingue qui traite partiellement de la textualité numérique à travers les problèmes d'éthique, "Technique et éthique des discours en ligne", dans la revue Linguas e Instrumentos Lingüisticos de l'Unicamp (Dias, Paveau 2016a) ; la même année est publié, également au Brésil, un dossier intitulé Análise do discurso digital: que conceitos e que dispositivos? (Analyse du discours numérique: quels concepts et quels dispositifs?), dans la revue Fragmentum, contenant plusieurs articles consacrés à l'écriture numérique et aux dispositifs iconotextuels (Dias, Paveau 2016b). Les différents collectifs dirigés par Fabien Liénard et Sami Zlitni, orientés plutôt vers la CMO (communication médiée par ordinateur) et la sociolinguistique, comportent cependant des travaux d'orientation discursive et textuelle qui participent de ce domaine de l'analyse des discours numériques natifs et plus largement des textialités (Zlitni, Liénard dir. 2013, Liénard dir. 2013, Liénard, Zlitni dir. 2015).

À ce propos, il faut également souligner un point qui ne sera pas abordé dans le présent dossier, mais qui est pris en compte par les chercheurs qui travaillent actuellement le texte numérique natif et plus largement l'écriture numérique: sa nature plurisémiotique à dominante iconique, ouverte au son, qu'il s'agisse de technographisme (Paveau 2017a, 2019 à par.), d'iconotexte (Goudet 2017, Muelle 2019 à par.), de photo-discours (Ghliss 2019 à par.), de ticker (Ruchon 2019 à par.), de vlog (Combe Celik 2016, 2017, 2019) ou de tout composant relevant de l'image fixe ou animée 
(Mayeur 2018, Ghliss, Jahjah 2019 sur la communication sur WhatsApp). Un précédent numéro hors-série de la revue Corela, consacré aux affordances (Ghliss, Perea, Ruchon dir. 2019), propose plusieurs articles sur cette nouvelle configuration du texte, iconisé de différentes manières, parce qu'il est composé, au sens presque typographique du terme, avec de l'image (mèmes, gifs, vignettes, etc.) ou parce qu'il est lui-même image (capture d'écran, photographie de texte).

\subsection{Autres disciplines}

Comme on l'a vu, le texte numérique natif ou textiel est largement traité du point de vue communicationnel en SIC depuis les années 1990. Ajoutons en guise d'exemple un collectif entièrement dédié à la question chez les chercheurs de Rennes 2 au début des années 2000, intitulé Écritures en ligne: pratiques et communautés (Chapelain dir. 2002), les réflexions de Valérie Jeanne Périer, d'Hélène Bourdeloie et de Jean Davallon sur les dispositifs d'écriture numérique (Jeanne Périer 2006, Bourdeloie 2012, Davallon 2012), les travaux de Marc Jahjah sur les annotations, qui coconstituent fondamentalement le texte numérique natif (Jahjah 2017a et b, 2018) ou les nombreux travaux effectués dans le cadre du projet PRECIP que synthétise Crozat et al. 2011. En littérature, les travaux sur les textes numériques ont également une antériorité : à partir des années 1990 en effet, plusieurs chercheurs développent à la fois des réflexions et des expérimentations sur la littérature numérique à partir des notions de textualité numérique et d'hypertextualité (Jean-Pierre Balpe et Serge Bouchardon sont par exemple à la fois auteurs et chercheurs). Citons par exemple Barbosa 1996, Vandendorpe 1999, Clément 2001, Bouchardon 2011, 2014a et b, Saemmer 2007, 2015a et b, pour ne mentionner que quelques titres au sein d'une production scientifique très importante depuis les années 1990. Dans ces travaux, c'est non seulement la nature même du texte qui est interrogée par la navigation hypertextuelle, mais également celle de la lecture, qui s'organise à partir des balises posées par le scripteur.

Dans le champ de la didactique des textes, la question de l'écriture numérique est prise en compte depuis le début des années 2010, les pratiques des élèves constituant évidemment un moteur et sans doute un outil de déverrouillage des obstacles vus plus haut pour ce type de recherche. C'est dans le cadre de l'étude de la littératie numérique que se développent la majeure partie des travaux. Citons, sans exhaustivité aucune, le collectif dirigé en 2012 par Monique Lebrun, Nathalie Lacelle et Jean-François Boutin, intitulé La littératie médiatique multimodale, qui insiste sur les compétences technologique et multimodale pour l'écriture numérique (Lebrun, Nacelle, Boutin eds 2012) ; les travaux de Marie-Claude Penloup $(2012,2017)$ et deux récents numéros du Français aujourd'hui consacré aux écritures numériques (Petitjean, Cordier, Alamargot dir. 2017, Petitjean, Brunel dir. 2018).

Dans un autre domaine, celui de l'édition, les recherches menées pour publier des textes en ligne - issus de l'imprimé tout d'abord, puis natifs de cet environnement - ont pareillement nécessité une meilleure compréhension de leurs unités constitutives et de leurs modes d'organisation. L'ouvrage de Marin Dacos et Pierre Mounier, L'édition électronique (2010), ouvre la réflexion sur ce que devrait être, compte tenu des possibilités du numérique, le texte électronique idéal (2010: 71 sqq): lisible, manipulable et citable. L'opérativité du texte numérique invite ainsi à la manipulation et à la circulation; il se voit par ailleurs éditorialisé, contextualisé dans une 
infrastructure qui lui confère une plus-value éditoriale (Ibid. 63). La notion d'éditorialisation, partie intégrante du textiel que ne permet pas d'appréhender le textuel, est reprise et travaillée par Marcello Vitali Rosati (2014, 2016, 2018; Vitali Rosati \& Sinatra 2014) qui lui confère le rôle de paratexte dynamique. Le récent colloque Écridil, qui a eu lieu à Montréal en 2018, sur le thème "Écrire, éditer, lire à l'ère numérique ", a permis une synthèse des réflexions actuelles sur ces questions : si la doxa médiatique et intellectuelle avance une faillite des publications numériques au profit d'un retour vers l'imprimé, les réalités des productions et créations montrent au contraire des transformations en profondeur de nos modes d'écriture de lecture et d'édition. Le colloque est publié sous la forme d'un livre liquide, c'est-à-dire collaboratif, transformable et annotable en ligne, sous le titre Versiono. Notes sur le livre numérique (ÉCRIDIL 2018).

\section{Présentation du numéro}

Sur la base de ces constats, nous avons proposé pour ce numéro plusieurs axes de questionnement en vue d'une exploitation du concept de textiel dans les recherches en sciences du texte et analyse (textuelle) des discours. Nous pensions en effet que cette notion avait été insuffisamment exploitée, y compris par leurs auteurs eux-mêmes, et qu'elle recelait pourtant des possibles théoriques et méthodologiques important pour une interrogation de la notion de textualité dans les contextes numériques natifs. La prise en compte de la textialité (impliquant donc une dimension de textualité technicisée [Jeanneret et al. (2003) 2013], qui ouvre le champ aux possibilités de manipulations) orienterait ainsi l'attention sur les spécificités du faire texte dans l'environnement numérique. Nous avons proposé aux auteurs de réfléchir par exemple aux questions suivantes, sans exclusive :

- Quel est le devenir du texte, tel qu'il est défini en contexte prénumérique, dans les environnements numériques natifs?

- Qu'est-ce qui "fait texte", dans les environnements numériques natifs? Quels seraient les "sentiments" du lecteur qui l'amènerait à porter un jugement de textualité ?

- Qui écrit le texte? Le scripteur, l'écrilecteur (le scripteur et le lecteur tout en même temps), la machine et ses algorithmes (avec quel degré d'autonomie) ?

- Qu'est-ce qui fonde la cohérence du texte numérique? Quels sont les opérateurs de textualisation à l'œuvre en régime numérique? Comment s'effectuent les liages textuels?

- Doit-on continuer à parler de texte pour les productions en ligne? À quelles conditions?

- Comment penser à nouveau frais les grandes questions liées à cette notion en linguistique textuelle, analyse du discours et sciences de l'information et de la communication : l'autonomie, la séquence, la cohérence, la cohésion, la continuité, le rapport écriture-lecture, l'intertextualité, le paratexte, la réception, la segmentation, l'unité textuelle, l'énonciation éditoriale, l'hypertexte, l'architexte, l'éditorialisation (liste ouverte)?

Les contributions reçues à la suite de ces propositions s'articulent autour de trois axes, selon qu'elles prennent pour objet les matières textielles, les processus d'écriture numérique ou les productions de textualités numériques.

La première section, matières textielles, s'ouvre sur une enquête visant à recontextualiser l'émergence du concept de textiel. Si l'on doit le terme exact à une 
trouvaille de Marie Després-Lonnet, celui-ci s'inscrit au demeurant dans une vaste dynamique de recherches collectives, interdisciplinaires et interuniversitaires, qui ont porté au devant de la scène la question du texte numérique et de ses usages. L'entretien mené avec Marie Després-Lonnet vise ainsi à préciser les acteurs de cette dynamique et le socle épistémologique dans lequel la notion s'est élaborée. Il met aussi en lumière la convergence d'un horizon de questionnements sur les propriétés agissantes du texte numérique comme textiel, sur le caractère central de son appropriation par l'usager mais aussi, plus largement, sur la pluralité des instances intervenant dans les processus de production du texte. Marcelo Vitali-Rosati opte de son côté pour une approche assez radicale, faisant du texte numérique l'architecture même de notre environnement. La théorie de l'éditorialisation, qu'il a formalisée en opérant la synthèse et en proposant le dépassement de conceptions antérieures (Vitali-Rosati 2016), suppose en effet que la mise en relation des textes dans l'écosystème connecté structure matériellement notre espace de vie. En ce sens, écrire le texte numérique reviendrait à agir concrètement sur l'espace dans lequel nous vivons. Dans leur article "L'énigme du textiel littéraire ", Clémence Jacquot et Marc Jahjah mettent en évidence ces diverses instances à l'œuvre dans l'actualisation du textiel. Par une étude de cas concrets, menée à partir d'un corpus d'œuvres littéraires issues du catalogue de la maison d'édition Publie.net, ils donnent à voir la genèse auctoriale et éditoriale d'une œuvre «textialisée ». L'analyse rend compte de l'épaisseur du textiel littéraire par une méthode combinant trois plans : la préfiguration, soit les cadres socio-discursifs anticipant son déploiement, la figuration, son apparition sur la scène éditoriale, et sa performance, soit le travail effectué par l'écrilecteur dans l'actualisation et l'interprétation du texte donné à lire.

La deuxième section, écritures numériques, place la focale sur les opérations de production du texte numérique et la complexité énonciative des écritures en ligne. À la question liminaire "Qui écrit le texte numérique?», Cléo Collomb et Samuel Goyet répondent en utilisant la notion de variabilité, issue des travaux de Marie-Anne Paveau sur les technodiscours, comme lieu d'observation de la part machinique, automatisée de l'écriture en ligne. Au départ de l'analyse sémiotique de d'interfaces web courantes, ils caractérisent ensuite ce que ce les énonciateurs non humains font à l'écriture du texte numérique. La troisième et dernière section, textualités numériques, porte plus précisément l'attention sur le faire texte, sur les processus qui permettent de poser sur le texte numérique un jugement de textualité au sens où l'entend Jean-Michel Adam (2015, cf. supra). À partir de l'étude d'un corpus de billets issus d'Hypothèses, plateforme de carnets de recherche en sciences humaines et sociales, Ingrid Mayeur interroge la manière dont le discours scientifique sur blog fait texte, dès lors que son unité se trouve mise en question par les possibilités de délinéarisation ouvertes par l'insertion de liens hypertextuels ou par les interactions générées en commentaires. Prenant appui sur la notion de gestes discursifs du savoir élaborée par le collectif liégeois Lttr13 (Lttr13 2016), elle propose de lire ces composantes du technodiscours des blogs scientifiques comme participant des processus de textualisation des discours de savoir. Émilie Née offre pour sa part une note de travail sur les pratiques d'écriture de la rédaction web (ou webrédaction): sur la base d'observations empiriques, nourries d'une expérience d'enseignement en milieu universitaire, elle propose des éléments de caractérisation de ces productions discursives susceptibles de servir des fins critiques mais, également, didactiques. La rédaction web s'accompagne en effet de discours d'escorte orientant la pratique, avec lesquels doivent composer les scripteurs afin de rédiger un texte numérique qui fasse sens aux yeux du lecteur, et qui anticipe adéquatement ses usages 
selon la sphère d'activité à laquelle il s'intègre - ce qu'illustrent des exemples pratiques mobilisés par l'auteure.

La postface rédigée par Jean-Michel Adam, que nous avons sollicité pour qu'il nous livre ses réactions aux textes du numéro, prend de la distance par rapport à la question des textualités numériques pour replacer le «faire texte » dans une perspective historique qui tient compte de "régimes sémio-médiolgiques " pluriels. Jean-Michel Adam est sensible aux analogies entre les traits des textes numériques natifs et les traits d'autres régimes d'expression, comme l'oralité, ce qui l'amène à adopter un point de vue différent et parfois critique sur les propositions du numéro. La discussion est donc ouverte, riche de futures investigations sur cette inépuisable question de ce qui fait texte, textuellement ou textiellement.

La présente livraison n'a en effet pas pour ambition de mettre à l'épreuve un cadre théorique abouti rendant compte des processus de textualisation dans les écosystèmes numériques: nous avons d'ailleurs souligné les limites de notre regard prenant essentiellement appui sur des travaux issus de la sphère scientifique francophone, mais aussi axé sur les éléments proprement langagiers du texte, dont le caractère polysémiotique s'accentue toutefois dans l'environnement numérique. Le faire texte numérique apparaît au demeurant comme une problématique originale et complexe dont nous entendons poser quelques jalons: nous formons le souhait que les contributions proposées au sein de ce numéro hors-série de la revue Corela contribuent à ouvrir des pistes d'exploration et de renouvellement des recherches en sciences du langage ${ }^{6}$.

\section{BIBLIOGRAPHIE}

N.B. : les liens ont été vérifiés le 15 juillet 2020

Adam Jean-Michel (2011). La linguistique textuelle: Introduction à l'analyse textuelle des discours, Paris, Armand Colin.

Adam Jean-Michel dir. (2015). Faire texte : frontières textuelles et opérations de textualisation. Besançon : Presses universitaires de Franche-Comté.

Adam Jean-Michel, Philippe Gilles, (2015). “Continuité et textualité”, dans Adam, Jean-Michel (dir.). 2015. Faire texte : frontières textuelles et opérations de textualisation. Besançon : Presses universitaires de Franche-Comté, p. 35-80.

Barbosa Pedro (1992). Metamorfoses do real. Criaçâo literaria e computador, Lisboa : Universidade nova de Lisboa.

Barbosa Pedro (1996). A Ciberliteratura. Criaçâo Literária e Computador, Lisboa : Cosmos.

Bonaccorsi, Julia. 2012. « Fantasmagories de l'écran: Pour une analyse visuelle de la textualité numérique ». HDR, Paris : Université Paris-Sorbonne.

Bouchardon Serge (2011). «Des figures de manipulation dans la création numérique », Protée 39, p. 37-46. 
Bouchardon Serge (2014a). «L'écriture numérique : objet de recherche et d'enseignement », Les Cahiers de la SFSIC 10, p. 225-235.

Bouchardon Serge (2014b) La valeur heuristique de la littérature numérique, Paris, Hermann.

Bourdeloie Hélène (2012), « L'appropriation des dispositifs d'écriture numérique : translittératie et capitaux culturel et social », Études de communication 38, http://edc.revues.org/3378

Chapelain Brigitte dir. (2002). Écritures en ligne : pratiques et communautés, actes du colloque de l'Université de Rennes 2. CERCOR (CERSIC), 26, 27 Septembre 2002, https:// archivesic.ccsd.cnrs.fr/sic_00126719/document

Chartier Roger (1993). « Le message écrit et des réceptions. Du codex à l'écran ». Revue des sciences morales et politiques 148 (2) : 295-313.

Chartier Roger (1997). Le livre en révolutions : Entretiens avec Jean Lebrun. Paris : Les Editions Textuel.

Chartier Roger (2001). « Lecteurs et lectures à l'âge de la textualité électronique ». Consulté le 27 mai 2016, http://cercamon.wikia.com/wiki/Chartier/

Lecteursetlectures\%C3\%A01\%27\%C3\%A2gedelatextualit\%C3\%A9\%C3\%A9lectronique

Chartier Roger (2006). « L'écrit sur l'écran. Ordre du discours, ordre des livres et manières de lire ». Entreprises et histoire 43, p. 15-25.

Chomsky Noam, 17.12.2010, « Interview with Noam Chomsky », Figure/Ground [site], http:// figureground.org/interview-with-noam-chomsky/

Chomsky Noam, 09.03.2011, « The secret of Noam : A Chomsky Interview », Brightest Young Things [site], http://brightestyoungthings.com/articles/the-secret-of-noam-a-chomsky-interview.htm

Cislaru G., Olive T. (2018), Le processus de textualisation. Analyse des unités linguistiques de performance écrite, Bruxelles : De Boeck.

Clément Jean (2001), « La littérature au risque du numérique », Document numérique. Volume X $\mathrm{n}^{\circ} \mathrm{X} / 2001$, pages 1 à X.

Combe Celik Christelle (2016). «Questions méthodologiques autour de l'étude de deux plateformes interactives multimodales : de la communauté de contenu à l'application intimiste », Línguas e Instrumentos Linguítiscos, p.211-230. 〈http://www.revistalinguas.com/edicao37/ artigo9.pdf

Combe Celik Christelle (2017). « Télécollaboration informelle 2.0 : le vlogue d'un américain en français sur YouTube ", Alsic 20-2, http://journals.openedition.org/alsic/3094

Combe Celik Christelle (2019). «Les genres numériques de la relation », Langage et société 167, p. 51-80.

Cormerais, Franck, et Jacques Athanase Gilbert (dirs.). 2016. Le texte à venir. Paris : Classiques Garnier.

Crozat Stéphane, Bachimont Bruno, Cailleau Isabelle, Bouchardon Serge, Gaillard Ludovic (201). «Éléments pour une théorie opérationnelle de l'écriture numérique », Document numérique, vol. 14/3, Paris : Hermès Lavoisier, p.9-33.

Dacos Marin, Mounier Pierre (2010). L'édition électronique, Paris : La Découverte.

Davallon, Jean, Marie Després-Lonnet, Yves Jeanneret, Joëlle Le Marec, et Emmanuël Souchier. (2013 [2003]). Lire, écrire, récrire : Objets, signes et pratiques des médias informatisés. Études et recherche. 
Paris : Éditions de la Bibliothèque publique d'information. http://books.openedition.org/ bibpompidou/394.

Davallon Jean (2012). L'économie des écritures sur le web/Volume 1 : traces d'usage dans un corpus de sites de tourisme. Paris : Lavoisier.

De Angelis Rossana (2018). « Textes et textures numériques », Signata $9 \underline{\mathrm{http}: / /}$

journals.openedition.org/signata/1675

Dias Cris, Paveau Marie-Anne dir. (2016a). Dossier « Técnica e ética dos discursos on-line » ( Technique et éthique des discours en ligne »), revue Linguas e Instrumentos Lingüisticos 37, http://www.revistalinguas.com/edicao37/edicao37.html

Dias Cristiane et Paveau Marie-Anne dir. (2016b). Dossier « Análise do discurso digital : que conceitos e que dispositivos? ", Fragmentum 48, https://periodicos.ufsm.br/fragmentum/issue/ view/1084

ECRIDIL (2018). Version0. Notes sur le livre numérique, actes du colloque ÉCRIDIL, Montréal, mai 2018.

Emerit Laetitia (2016). «La notion de lieu de corpus : un nouvel outil pour l'étude des terrains numériques en linguistique ». Corela. Cognition, représentation, langage, no 14-1 (juin). doi:10.4000/ corela.4594

Ghliss Yosra à par. (2019). « Les photo-discours via WhatsApp : de l'affordance mobile à l'agentivité affective », Corela Hors Série 28, dossier « Les affordances langagières : textualité numérique, matérialité discursive ».

Ghliss Yosra, Jahjah Marc (2019). « Habiter Whatsapp. Éléments d'analyse postdualiste des interactions en espace numérique ", Langage et société 167, p. 29-50.

Ghliss Yosra, Perea François et Catherine Ruchon dir. (2019) à par. Dossier « Les affordances langagières : textualité numérique, matérialité discursive", Corela Hors Série 28.

Goudet Laura-Gabrielle (2017). « Le dispositif iconotextuel des mèmes : clichés variables et subversion des genres », Fragmentum 48, https://periodicos.ufsm.br/fragmentum/article/view/ 23294

Habert Benoît (2005). « Portrait de linguiste(s) à l'instrument », Texto! [en ligne], vol. X, n4 , http://www.revue-texto.net/Corpus/Publications/Habert/Habert_Portrait.html

Jahjah Marc (2017a). « Des énoncés sans énonciateur? Du surlignement à citation dans le dispositif Kindle d'Amazon », Semen 41, http://semen.revues.org/10587

Jahjah Marc (2017b). « “Annoter le monde et améliorer l'humanité” : imaginaires et fabrication d'un logiciel d'annotation ", dans Gérald Kembellec et Evelyne Broudoux (dir.), Ecrilecture augmentée dans les communautés scientifiques, ISTE éditions, p. 71-88.

Jahjah Marc (2018). «Culture imprimée et culture numérique : au-delà de Gutenberg, les enjeux du "texte livresque" ", La Revue de la Bibliothèque nationale universitaire de Strasbourg, hors-série Gutenberg, p.90-99.

Jeanne Perrier Valérie (2006). « Des outils d'écriture aux pouvoirs exorbitants ?» Réseaux 137, p. 97-131.

Jeanneret, Yves, Annette Béguin, Dominique Cotte, Sarah Labelle, Valérie Perrier, Philippe Quinton, et Emmanuël Souchier. (2013 [2003]). « Chapitre II. Formes observables, représentations et appropriation du texte de réseau ». In Lire, écrire, récrire: Objets, signes et pratiques des médias informatisés, par Jean Davallon, Marie Després-Lonnet, et Joëlle Le Marec, 93-158. Études et 
recherche. Paris : Éditions de la Bibliothèque publique d'information. http://

books.openedition.org/bibpompidou/412.

Jeanneret Yves, et Emmanuël Souchier (2005). «L'énonciation éditoriale dans les écrits d'écran ». Communication et langages 145 (1): 3-15. doi:10.3406/colan.2005.3351.

Lebrun Monique, Lacelle Nathalie \& Jean-François Boutin eds. (2012). La littératie médiatique multimodale: de nouvelles approches en lecture-écriture à l'école et hors de l'école, Québec: Presses de l'Université du Québec.

Lefebvre Julie, Léon Jacqueline, Puech Christian dir., (2016). Dossiers d'HEL n ${ }^{\circ} 9$ : Écriture(s) et représentations du langage et des langues, Actes du colloque SHESL-HTL 2013, 25-26 janvier 2013, Paris, https://halshs.archives-ouvertes.fr/halshs-01282506

Liénard Fabien dir. (2013). Dossier « Culture, identité et écritures numériques ». Epistémè.

Liénard Fabien, Zlitni Sami dir., (2015). La communication électronique : enjeux, stratégies, opportunités, Limoges : Lambert-Lucas.

Lieutard A. org. (2019). Minuscules et capitales. Systèmes graphiques des langues de France et d'ailleurs, colloque Université Paul-Valéry, 4 et 5 avril.

Lttr13 [pseud. Provenzano, François, Stéphane Polis et Sémir Badir] (2016). « Figures de l'énonciation : les gestes discursifs du savoir ». In Figures en discours, édité par Amir Biglari et Geneviève Salvan, 93-116. Louvain-la-Neuve : Academia - L'Harmattan. https://orbi.uliege.be/ handle/2268/194724.

Mayaffre Damon (2007). «Philologie et/ou herméneutique numérique : nouveaux concepts pour de nouvelles pratiques ", in Rastier François, Ballabriga Michel (éd.), Corpus en Lettres et Sciences sociales. Des documents numériques à l'interprétation, Toulouse : PUT, p. 15-26.

Mayeur Ingrid (2016). « Textiel et technodiscours : sur l'articulation des traits langagiers et techniques au sein des écrits numériques », Dériv@tions [carnet de recherche], https:// driv.hypotheses.org/164

Mayeur Ingrid (2018 [2019] si à paraître dans Sens public). « Le discours scientifique en SHS au prisme de sa matérialité langagière. Enrichissement hypertextuel et intertexte des carnets de recherche en ligne », Dériv@tions [carnet de recherche], https://driv.hypotheses.org/ 650\#more-650

Mayeur Ingrid (2019). Recherches en cours. Discours de savoir numériques et actualité scientifique dans les carnets de recherche de la plateforme Hypothèses, thèse de doctorat présentée sous la direction de MM. B.-O. Dozo et F. Provenzano, Université de Liège, http://hdl.handle.net/ $2268 / 232256$

Muelle Léo (2019) à par., « La puissance du phototexte sur le Web 2.0 : identité, visibilité et militantisme », in Foucher Charlotte, Nachtergael Magali, (dir.), Le Phototexte engagé. Du militantisme aux luttes de visibilité, actes du colloque de Saint-Denis, MSH Paris-Nord, mai 2018. Page Ruth (2012). « The Linguistics of Self Branding and Micro-celebrity in Twitter: The Role of Hashtags », Discourse and Communication 6 (2), p.181-201.

Paveau Marie-Anne (2013). «Technodiscursivités natives sur Twitter. Une écologie du discours numérique », Epistémé, p.139-176.

Paveau, Marie-Anne (2015a). « En naviguant en écrivant. Réflexions sur les textualités numériques ». In Faire texte. Frontières textuelles et opérations de textualisation, par Jean-Michel Adam. https://hal-univ-paris13.archives-ouvertes.fr/hal-01163507/document. 
Paveau, Marie-Anne (2015b). «Présentation. Les textes numériques sont-ils des textes?» Itinéraires. Littérature, textes, cultures, no 2014-1 (février). https://itineraires.revues.org/2312.

Paveau Marie-Anne dir. (2015). Dossier « Textualités numériques », Itinéraires. Littérature, textes, cultures, https://journals.openedition.org/itineraires/2258

Paveau, Marie-Anne (2016). « Des discours et des liens. Hypertextualité, technodiscursivité, écrilecture ». Semen 42 , p. 23-48.

Paveau, Marie-Anne (2017a). L'analyse du discours numérique. Dictionnaire des formes et des pratiques, Paris : Hermann.

Paveau Marie-Anne (2017b). « Du microscope à la caméra subjective. Les observables natifs de l'internet », Le discours et la langue 9.2., p.160-172.

Paveau Marie-Anne (2019) à par. « Technographismes en ligne. Énonciation matérielle visuelle et iconisation du texte », dans Yosra Ghliss, François Perea et Catherine Ruchon (dir.), dossier « Les affordances langagières : textualité numérique, matérialité discursive », Corela HS 28.

Penloup Marie-Claude (2012). «Littératies numériques : quels enjeux pour la didactique de l'écriture-lecture? », Les Cahiers de l'Acedle, 9(2), p.129-140, http://acedle.org/old/IMG/pdf/ 06_Penloup.pdf

Penloup Marie-Claude (2017). « Didactique de l'écriture : le déjà-là des pratiques d'écriture numérique ", Le français aujourd'hui 196, p.57-70, https://www.cairn.info/revue-le-francaisaujourd-hui-2017-1-page-57.htm

Petitjean Anne-Marie, Cordier Anne et Denis Alamargot dir. (2017). Dossier « Écriture numérique : des usages sociaux aux formations ", Le français aujourd'hui 196.

Petitjean Anne-Marie, Brunel Magali dir. (2018). Dossier « Écriture numérique : la conversion du littéraire? ", Le français aujourd'hui 200.

Rastier, François (2001). Arts et sciences du texte. Paris : Presses Universitaires de France.

Rastier François (2007). « Passages », Corpus 6, http://journals.openedition.org/corpus/832

Rastier François (2011). La mesure et le grain. Sémantique de corpus. Paris : Honoré Champion.

Ruchon Catherine (2019) à par., « De l'affordance injonctive à la créativité discursive : l'exemple du ticker numérique », Corela Hors Série 28, dossier « Les affordances langagières : textualité numérique, matérialité discursive ».

Saemmer Alexandra (2007). Matières textuelles sur support numérique. Université de Saint-Etienne.

Saemmer Alexandra (2015a). Rhétorique du texte numérique : figures de la lecture, anticipations de pratiques, Lyon : Presses de l'Enssib.

Saemmer, Alexandra (2015b). « Hypertexte et narrativité », Critique 819-820 (8): 637-52.

Simon Justine dir (2016). Dossier « Le discours hypertextualisé. Problématique de renouvellement des pratiques d'écriture et de lecture », Semen 42.

Simon Justine dir. (2018). Le discours hypertextualisé - Espaces énonciatifs mosaïques, Besançon :

Presses universitaires de Franche-Comté.

Simone Rafaele (2012). Pris dans la toile. L'esprit au temps du web, trad. de l'italien par G. Larcher, Paris : Gallimard.

Souchier Emmanuël (1996). « L'écrit d'écran, pratiques d'écriture \& informatique ».

Communication et langages 107 (1): 105-19. doi:10.3406/colan.1996.2662 
Souchier Emmanuël (1998). « L'image du texte : pour une théorie de l'énonciation éditoriale ». Les cahiers de médiologie 6 (2): 137-45

Souchier, Emmanuël, Jeanneret Yves, Le Marec Joëlle dir. (2003.) Lire, écrire, récrire. Objets, signes et pratiques des médias informatisés, Paris, BPI.

Souchier, Emmanuël dir. (2004). « Du "document numérique" au "textiel” » [dossier].

Communication et langages, ${ }^{\circ}$ 140, http://www.persee.fr/issue/colan0336-15002004num1401

Souchier, Emmanuël (2016). « Le grand entretien avec Emmanuël Souchier ». In Le texte à venir, édité par Franck Cormerais et Jacques Athanase Gilbert, 189-212. Paris : Classiques Garnier.

Tardy Cécile, et Yves Jeanneret (2007). L'écriture des médias informatisés : espaces de pratiques. Paris : Hermès science.

Vandendorpe Christian (1999). Du papyrus à l'hypertexte. Essai sur les mutations du texte et de la lecture, Montréal : Boréal/Paris : La Découverte.

Vitali-Rosati, Marcello et Michael E. Sinatra, éd. (2014). Pratiques de l'édition numérique. Parcours numérique. Montréal : Presses de l'Université de Montréal. http://books.openedition.org/pum/ 306

Vitali Rosati Marcello (2016). « What is editorialization? » Sens public, janvier. http://www.senspublic.org/article1059.html

Vitali-Rosati, Marcello. (2018). On Editorialization: Structuring Space and Authority in the Digital Age. Theory on Demand 26. Amsterdam: Institute of Network Cultures. http://networkcultures.org/ blog/publication/tod-26-on-editorialization-structuring-space-and-authority-in-the-digitalage/.

Zlitni Sami et Liénard Fabien dir. (2013). La communication électronique en questions, Berne : Peter Lang.

\section{NOTES}

1. Natif dans cette perspective veut dire produits originellement au sein de ces espaces connectés, et désigne des textualités, ou, on le verra, des textialités, qui n'existent pas hors de ces espaces.

2. Pour des raisons d'agenda de travail, Emmanuël Souchier a malheureusement dû décliner notre proposition de collaboration à ce numéro; le caractère récent de cette source rend toutefois son absence moins dommageable.

3. Champ de l'herméneutique du texte ouvert par Rastier 2011 ou de la philologie numérique proposé par Mayaffre 2007, qui sont les références principales de la majeure partie des chercheur.e.s qui travaillent dans ce domaine.

4. François Rastier préconise dans Art et sciences du texte le recours à une philologie numérique adaptée aux nouvelles réalités matérielles des textes en circulation (Rastier 2001). L'objet de la démarche vise la constitution de corpus fiables; Rastier rappelle à cet endroit le caractère construit de tout texte, nécessitant un regard critique sur ses modes d'établissement. Il insiste à cette occasion sur la délinéarisation du texte numérique : celle-ci établit une relation nouvelle entre le texte et l'intertexte, qui doivent désormais être embrassés d'un seul regard. Dans la conception de Rastier, la délinéarisation est alors définie comme extraction du fil de l'énoncé, et ne recoupe pas celle, d'une autre nature, qu'accomplit selon Marie-Anne Paveau le lien hypertexte en environnement connecté (Paveau 2017 et infra) : « De manière plus rassise, dans le domaine de la recherche d'information, la linguistique de corpus trouve ses finalités dans des 
applications qui lui permettent de favoriser l'interprétation en extrayant des passages susceptibles d'être pertinents en fonction d'une tâche déterminée. La conceptualisation du passage lui est particulièrement nécessaire, dans la mesure où la recherche d'informations impose une délinéarisation des textes et une hiérarchisation sélective des extraits » (Rastier $2007: 43)$.

5. Nous entendons par étude écologique l'analyse des énoncés dans leur environnement natif, prenant en compte l'ensemble des paramètres de production : les écrilecteurs, les phénomènes d'audience (c'est-à-dire la représentation des destinataires, très complexe sur les réseaux sociaux comme Twitter ou Facebook, voir à ce propos Page 2012), les dispositifs logiciels d'écriture (écrire sur un blog, un site de presse ou un réseau social sont des choses différentes), les formats préconstruits, les types d'appareil (l'écriture numérique et donc les textes produits ne sont pas les mêmes sur un smartphone et un ordinateur par exemple), les types de navigateur (c'est différent de produire des textes en utilisant des navigateurs classiques comme Firefox, Safari ou Chrome et d'écrire à partir de Tor qui est crypté, et ne produit donc pas les mêmes configurations algorithmiques).

6. Les articles de ce numéro ont été écrits au printemps 2019, soit environ un an et demi avant leur publication. La recherche étant très rapide sur cette question, il est possible que cette livraison accuse quelques manques, que les lecteurs et lectrices spécialistes ne manqueront pas de combler.

\section{AUTEURS}

\section{INGRID MAYEUR}

Université de Liège

\section{MARIE-ANNE PAVEAU}

Université Sorbonne Paris Nord 